Pacific

Journal of

Mathematics

COHOMOLOGY OF COMPLETE INTERSECTIONS IN TORIC VARIETIES

Anvar R. Mavlyutov

Volume $191 \quad$ No. 1

November 1999 


\title{
COHOMOLOGY OF COMPLETE INTERSECTIONS IN TORIC VARIETIES
}

\author{
Anvar R. MAVLyutov
}

\begin{abstract}
We explicitly describe cohomology of complete intersections in compact simplicial toric varieties.
\end{abstract}

In this paper we will study intersections of hypersurfaces in compact simplicial toric varieties $\mathbf{P}_{\Sigma}$. The main purpose is to relate naturally the Hodge structure of a complete intersection $X_{f_{1}} \cap \ldots \cap X_{f_{s}}$ in $\mathbf{P}_{\Sigma}$ to a graded ring. Originally this idea appears in $[\mathbf{G r}],[\mathbf{S t}],[\mathbf{D o l}],[\mathbf{P S}]$. The case of a hypersurface in a toric variety has been treated in $[\mathbf{B C}]$. Also the Hodge structure of complete intersections in a projective space was described in $[\mathbf{T e}],[\mathbf{K o}]$, $[\mathbf{L}],[\mathbf{D i}],[\mathbf{N a}]$. The common approach was to reduce studying of the Hodge structure on a complete intersection to studying of the Hodge structure on a hypersurface in a higher dimensional projective variety. This is the idea of a "Cayley trick". About a Cayley trick in the toric context see [GKZ], $[\mathbf{D K}],[\mathbf{B B}]$. A special case of a complete intersection (when it is empty) in a complete simplicial toric variety was elaborated in $[\mathbf{C C D}]$. The basic references on toric varieties are $[\mathbf{F} \mathbf{1}],[\mathbf{O}],[\mathbf{D a}],[\mathbf{C}]$.

The paper is organized as follows:

Section 1 establishes notation and studies cohomology of subvarieties in a complete simplicial toric variety. In Section 2 we describe a Cayley trick for toric varieties. In Section 3 we prove the main result where we relate the Hodge components $H^{d-s-p, p}\left(X_{f_{1}} \cap \cdots \cap X_{f_{s}}\right)$ in the middle cohomology group to homogeneous components of a graded ring. Section 4 treats a special case of complete intersections: a nondegenerate intersection.

Acknowledgment. I would like to thank D. Cox for his advice and useful comments.

\section{Quasi-smooth intersections.}

We first fix some notation. Let $M$ be a lattice of $\operatorname{rank} d, N=\operatorname{Hom}(M, Z)$ the dual lattice; $M_{\mathbf{R}}$ (resp. $N_{\mathbf{R}}$ ) denotes the $\mathbf{R}$-scalar extension of $M$ (resp. of $N$ ). Let $\Sigma$ be a rational simplicial complete $d$-dimensional fan in $N_{\mathbf{R}}$ $[\mathbf{B C}], \mathbf{P}_{\Sigma}$ a complete simplicial toric variety associated with this fan.

Such a toric variety can be described as a geometric quotient $[\mathbf{C}]$. Let $S(\Sigma)=\mathbf{C}\left[x_{1}, \ldots, x_{n}\right]$ be the polynomial ring over $\mathbf{C}$ with variables $x_{1}, \ldots, x_{n}$ 
corresponding to the integral generators $e_{1}, \ldots, e_{n}$ of the 1-dimensional cones of $\Sigma$. For $\sigma \in \Sigma$ let $\hat{x}_{\sigma}=\prod_{e_{i} \notin \sigma} x_{i}$, and let $B(\Sigma)=\left\langle\hat{x}_{\sigma}: \sigma \in \Sigma\right\rangle \subset S$ be the ideal generated by the $\hat{x}_{\sigma}$ 's. This ideal gives the variety $Z(\Sigma)=$ $\mathbf{V}(B(\Sigma)) \subset \mathbf{A}^{n}$. The toric variety $\mathbf{P}=\mathbf{P}_{\Sigma}$ will be a geometric quotient of $U(\Sigma):=\mathbf{A}^{n} \backslash Z(\Sigma)$ by the group $\mathbf{D}:=\operatorname{Hom}_{\mathbf{Z}}\left(A_{d-1}(\mathbf{P}), \mathbf{C}^{*}\right)$, where $A_{d-1}(\mathbf{P})$ is the Chow group of Weil divisors modulo rational equivalence.

Each variable $x_{i}$ in the coordinate ring $S(\Sigma)$ corresponds to a torusinvariant irreducible divisor $D_{i}$ of $\mathbf{P}$. As in $[\mathbf{C}]$, we grade $S=S(\Sigma)$ by assigning to a monomial $\prod_{i=1}^{n} x_{i}^{a_{i}}$ its degree $\left[\sum_{i=1}^{n} a_{i} D_{i}\right] \in A_{d-1}(\mathbf{P})$. A polynomial $f$ in the graded piece $S_{\alpha}$ corresponding to $\alpha \in A_{d-1}(\mathbf{P})$ is said to be $\mathbf{D}$-homogeneous of degree $\alpha$.

Let $f_{1}, \ldots, f_{s}$ be $\mathbf{D}$-homogeneous polynomials. They define a zero set $\mathbf{V}\left(f_{1}, \ldots, f_{s}\right) \subset \mathbf{A}^{n}$, moreover $\mathbf{V}\left(f_{1}, \ldots, f_{s}\right) \cap U(\Sigma)$ is stable under the action of $\mathbf{D}$ and hence descends to a closed subset $X \subset \mathbf{P}$, because $\mathbf{P}$ is a geometric quotient.

Definition 1.1. We say that $X$ is a quasi-smooth intersection if $\mathbf{V}\left(f_{1}, \ldots\right.$, $\left.f_{s}\right) \cap U(\Sigma)$ is either empty or a smooth subvariety of codimension $s$ in $U(\Sigma)$.

Remark 1.2. This notion generalizes a nonsingular complete intersection in a projective space. Notice that since the $(n-d)$-dimensional group $\mathbf{D}$ has only zero dimensional stabilizers $[\mathbf{B C}], X$ is of pure dimension $d-s$ or empty.

We can now relate this notion to a V-submanifold (see Definition 3.2 in $[\mathrm{BC}])$.

Proposition 1.3. If $X \subset \mathbf{P}$ is a closed subset of codimension s defined by D-homogeneous polynomials $f_{1}, \ldots, f_{s}$, then $X$ is a quasi-smooth intersection if and only if $X$ is a $V$-submanifold of $\mathbf{P}$.

The proof of this is very similar to the proof of the Proposition 3.5 in $[\mathrm{BC}]$.

The next result is a Lefschetz-type theorem.

Proposition 1.4. Let $X \subset \mathbf{P}$ be a closed subset, defined by $\mathbf{D}$-homogeneous polynomials $f_{1}, \ldots, f_{s}$, in a complete simplicial toric variety $\mathbf{P}$. If $f_{1}, \ldots$, $f_{s} \in B(\Sigma)$, then the natural map $i^{*}: H^{i}(\mathbf{P}) \rightarrow H^{i}(X)$ is an isomorphism for $i<d-s$ and an injection for $i=d-s$. In particular, this is valid if $X$ is an intersection of ample hypersurfaces.

Proof. We can present $X=X_{f_{1}} \cap \ldots \cap X_{f_{s}}$, where $X_{f_{i}} \subset \mathbf{P}$ is a hypersurface defined by $f_{i}$. As it was shown in the proof of the Proposition 10.8 [BC], if $f \in B(\Sigma)$ then $\mathbf{P} \backslash X_{f}=\left(\mathbf{A}^{n} \backslash \mathbf{V}(f)\right) / \mathbf{D}(\Sigma)$ is affine, hence $H^{i}\left(\mathbf{P} \backslash X_{f}\right)=0$ for $i>d$. We will prove by induction on $s$ that $H^{i}\left(\mathbf{P} \backslash\left(X_{f_{1}} \cap \ldots \cap X_{f_{s}}\right)\right)=0$ for $i>d+s-1$. Consider the Mayer-Vietoris sequence $\cdots \rightarrow H^{i}(U \cap V) \rightarrow H^{i+1}(U \cup V) \rightarrow H^{i+1}(U) \oplus H^{i+1}(V) \rightarrow H^{i+1}(U \cap V) \rightarrow \cdots$ 
with $U=\mathbf{P} \backslash\left(X_{f_{1}} \cap \ldots \cap X_{f_{s-1}}\right), V=\mathbf{P} \backslash X_{f_{s}}$. Notice that $U \cup V=\mathbf{P} \backslash\left(X_{f_{1}} \cap\right.$ $\left.\ldots \cap X_{f_{s}}\right)$ and $U \cap V=\cup_{i=1}^{s-1} \mathbf{P} \backslash\left(X_{f_{i}} \cup X_{f_{s}}\right)=\mathbf{P} \backslash\left(X_{f_{1} \cdot f_{s}} \cap \ldots \cap X_{f_{s-1} \cdot f_{s}}\right)$. So, using the induction and the above sequence, we obtain that $H^{i}(\mathbf{P} \backslash X)=0$ for $i>d+s-1$. As a consequence of this, $X$ is nonempty unless $s>d$ because the dimension $h^{2 d}(\mathbf{P})=1$. Since $\mathbf{P} \backslash X$ is a V-manifold, Poincaré duality implies that $H_{c}^{i}(\mathbf{P} \backslash X)=0$ for $i \leq d-s$. Now the desired result follows from the long exact sequence of the cohomology with compact supports $(X$ and $\mathbf{P}$ are compact):

$$
\cdots \rightarrow H_{c}^{i}(\mathbf{P} \backslash X) \rightarrow H_{c}^{i}(\mathbf{P}) \rightarrow H_{c}^{i}(X) \rightarrow H_{c}^{i+1}(\mathbf{P} \backslash X) \rightarrow H_{c}^{i+1}(\mathbf{P}) \rightarrow \cdots
$$

If $X$ is an intersection of ample hypersurfaces defined by $f_{1}, \ldots, f_{s}$, then Lemma $9.15[\mathbf{B C}]$ gives us that $f_{1}, \ldots, f_{s}$ belong to $B(\Sigma)$.

Corollary 1.5. A quasi-smooth intersection $X=X_{f_{1}} \cap \ldots \cap X_{f_{s}}$, defined by $f_{1}, \ldots, f_{s} \in B(\Sigma)$, has pure dimension $d-s$.

Since the dimension of $H^{0}(X, \mathbf{C})$ is the number of connected components of $X$, we obtain another important result.

Corollary 1.6. An intersection $X_{f_{1}} \cap \ldots \cap X_{f_{s}}$, defined by $f_{1}, \ldots, f_{s} \in$ $B(\Sigma)$, in a complete simplicial toric variety $\mathbf{P}_{\Sigma}$ is connected provided $s<$ $\operatorname{dim} \mathbf{P}_{\Sigma}$.

Remark 1.7. If the polynomials $f_{1}, \ldots, f_{s}$ have ample degrees, then this corollary follows from a more general statement in [FL1] (see also [FL2] and $[\mathbf{F H}]$ for connectedness theorems).

\section{2. "Cayley trick".}

We will explore a Cayley trick to reduce studying of the cohomology of quasi-smooth intersections to results already known for hypersurfaces.

Let $L_{1}, \ldots, L_{s}$ be line bundles on a complete $d$-dimensional toric variety $\mathbf{P}=\mathbf{P}_{\Sigma}$, and let $\pi: \mathbf{P}(E) \rightarrow \mathbf{P}$ be the projective space bundle associated to the vector bundle $E=L_{1} \oplus \cdots \oplus L_{s}$. Then the $\mathbb{P}^{s-1}$-bundle $\mathbf{P}(E)$ is a toric variety. The fan corresponding to it can be described as follows $[\mathbf{O}$, p. 58]. Suppose that support functions $h_{1}, \ldots, h_{s}$ give rise to the isomorphism classes of line bundles $\left[L_{1}\right], \ldots,\left[L_{s}\right] \in \operatorname{Pic}(\mathbf{P})$, respectively. Introduce a Z Z-module $N^{\prime}$ with a Z-basis $\left\{n_{2}, \ldots, n_{s}\right\}$ and let $\tilde{N}:=N \oplus N^{\prime}$ and $n_{1}:=-n_{2}-\cdots-n_{s}$. Denote by $\tilde{\sigma}$ the image of each $\sigma \in \Sigma$ under the R-linear map $N_{\mathbf{R}} \rightarrow \tilde{N}_{\mathbf{R}}$ which sends $y \in N_{\mathbf{R}}$ to $y-\sum_{j=1}^{s} h_{j}(y) n_{j}$. On the other hand, let $\sigma_{i}^{\prime}$ be the cone in $N_{\mathbf{R}}^{\prime}$ generated by $n_{1}, \ldots, n_{i}, n_{i+1}, \ldots, n_{s}$ and let $\Sigma^{\prime}$ be the fan in $N_{\mathbf{R}}^{\prime}$ consisting of the faces of $\sigma_{1}^{\prime}, \ldots, \sigma_{s}^{\prime}$. Then $\mathbf{P}(E)$ corresponds to the fan $\tilde{\Sigma}:=\left\{\tilde{\sigma}+\sigma^{\prime}: \sigma \in \Sigma, \sigma^{\prime} \in \Sigma^{\prime}\right\}$. From this description it is easy to see that if $\Sigma$ is a complete simplicial fan then $\mathbf{P}\left(L_{1} \oplus \cdots \oplus L_{s}\right)$ is a complete simplicial toric variety. We see that the integral generators of 
the 1-dimensional cones in $\tilde{\Sigma}$ are given by

$$
\begin{aligned}
\tilde{e}_{i} & =e_{i}-\sum_{1 \leq j \leq s} h_{j}\left(e_{i}\right) n_{j}, \quad i=1, \ldots n, \\
\tilde{n}_{1} & =-n_{2}-\cdots-n_{s}, \\
\tilde{n}_{j} & =n_{j}, \quad j=2, \ldots, s,
\end{aligned}
$$

where $e_{1}, \ldots, e_{n}$ are the integral generators of the 1-dimensional cones in $\Sigma$.

The homogeneous coordinate ring of $\mathbf{P}(E)$ is the polynomial ring

$$
R=\mathbf{C}\left[x_{1}, \ldots, x_{n}, y_{1}, \ldots, y_{s}\right],
$$

where $x_{i}$ corresponds to $\tilde{e}_{i}$ and $y_{j}$ corresponds to $\tilde{n}_{j}$. This ring has a grading by the Chow group $A_{d+s-2}(\mathbf{P}(E))$. Since $\mathbf{P}$ is a normal variety, there is an embedding of the Picard group $\operatorname{Pic}(\mathbf{P}) \hookrightarrow A_{d-1}(\mathbf{P})$. We want to show that if some polynomials $f_{j} \in S(\Sigma)=\mathbf{C}\left[x_{1}, \ldots, x_{n}\right]$ have the property $\operatorname{deg}\left(f_{j}\right)=$ $\left[L_{j}\right] \in \operatorname{Pic}(\mathbf{P})$, then the polynomials $y_{j} f_{j}$ all have the same degree in $R$. This will allow us to consider a hypersurface defined by the homogeneous polynomial $F=\sum_{j=1}^{s} y_{j} f_{j}$.

Lemma 2.1. Let $f_{1}, \ldots, f_{s} \in S(\Sigma)$ be D-homogeneous polynomials, such that $\operatorname{deg}\left(f_{j}\right)=\left[L_{j}\right]$ for some line bundles $L_{1}, \ldots, L_{s}$. Then $F=\sum_{j=1}^{s} y_{j} f_{j}$ is homogeneous in $R$ and its degree is the isomorphism class $\left[O_{\mathbf{P}(E)}(1)\right]$ of the canonical line bundle on $\mathbf{P}(E)=\mathbf{P}\left(L_{1} \oplus \cdots \oplus L_{s}\right)$.

Proof. To prove that $F$ is a homogeneous polynomial we will repeat the arguments in the proof of Lemma 3.5 in $[\mathbf{C C D}]$. Let $D_{1}, \ldots, D_{n}$ be the torus-invariant divisors on $\mathbf{P}=\mathbf{P}_{\Sigma}$ corresponding to the 1-dimensional cones of the fan $\Sigma$. Then the pullback $\pi^{*} D_{i}$ is the torus-invariant divisor of $\mathbf{P}(E)$ corresponding to the cone generated by $\tilde{e}_{i}$. Also denote by $D_{j}^{\prime}$ the torusinvariant divisor corresponding to $\tilde{n}_{j}$. Let $\tilde{M}=M \oplus M^{\prime}$ be the lattice dual to $\tilde{N}=N \oplus N^{\prime}$ with $M^{\prime}=\operatorname{Hom}\left(N^{\prime}, \mathbf{Z}\right)$ having $\left\{n_{2}^{*}, \ldots, n_{s}^{*}\right\}$ as a basis dual to $\left\{n_{2}, \ldots, n_{s}\right\}$. The divisor corresponding to the character $\chi^{n_{j}^{*}}$ is

$$
\begin{aligned}
\operatorname{div}\left(\chi^{n_{j}^{*}}\right) & =\sum_{i=1}^{n}\left\langle n_{j}^{*}, \tilde{e}_{i}\right\rangle \pi^{*} D_{i}+\sum_{k=1}^{s}\left\langle n_{j}^{*}, \tilde{n}_{k}\right\rangle D_{k}^{\prime} \\
& =\sum_{i=1}^{n}\left(h_{1}\left(e_{i}\right)-h_{j}\left(e_{i}\right)\right) \pi^{*} D_{i}-D_{1}^{\prime}+D_{j}^{\prime} .
\end{aligned}
$$

Therefore, $\left[D_{j}^{\prime}\right]+\left[\pi^{*} L_{j}\right]$ all have the same degree in the Chow group $A_{d+s-2}(\mathbf{P}(E))$, and, consequently, $F$ is a homogeneous polynomial.

Now consider the following exact sequence $[\mathbf{M}]$ :

$$
0 \rightarrow O_{\mathbf{P}(E)} \rightarrow \pi^{*} E^{*} \otimes O_{\mathbf{P}(E)}(1) \rightarrow \mathrm{T}_{\mathbf{P}(E)} \rightarrow \pi^{*} \mathrm{~T}_{\mathbf{P}} \rightarrow 0,
$$


where $\mathrm{T}_{X}$ denotes the tangent bundle, $E^{*}$ is the dual bundle. From here we can compute the Chern class

$$
\begin{aligned}
c_{1}\left(\mathrm{~T}_{\mathbf{P}(E)}\right) & =c_{1}\left(\pi^{*} \mathrm{~T}_{\mathbf{P}}\right)+c_{1}\left(\pi^{*} E^{*} \otimes O_{\mathbf{P}(E)}(1)\right) \\
& =\pi^{*} c_{1}\left(\mathrm{~T}_{\mathbf{P}}\right)-\pi^{*} c_{1}(E)+s \cdot c_{1}\left(O_{\mathbf{P}(E)}(1)\right) .
\end{aligned}
$$

Hence, $s \cdot c_{1}\left(O_{\mathbf{P}(E)}(1)\right)=\pi^{*} c_{1}\left(L_{1}\right)+\cdots+\pi^{*} c_{1}\left(L_{s}\right)+c_{1}\left(\mathrm{~T}_{\mathbf{P}(E)}\right)-\pi^{*} c_{1}\left(\mathrm{~T}_{\mathbf{P}}\right)$. On the other hand, from the generalized Euler exact sequence [BC, $\S 12]$ we get

$$
0 \rightarrow O_{\mathbf{P}}^{n-d} \rightarrow \oplus_{i=1}^{n} O_{\mathbf{P}}\left(D_{i}\right) \rightarrow \mathrm{T}_{\mathbf{P}} \rightarrow 0 .
$$

This implies that $c_{1}\left(\mathrm{~T}_{\mathbf{P}}\right)=\left[D_{1}\right]+\cdots+\left[D_{n}\right]$. Similarly we have $c_{1}\left(\mathrm{~T}_{\mathbf{P}(E)}\right)=$ $\left[\pi^{*} D_{1}\right]+\cdots+\left[\pi^{*} D_{n}\right]+\left[D_{1}^{\prime}\right]+\cdots+\left[D_{s}^{\prime}\right]$. Under the identification $\operatorname{Pic}(\mathbf{P}(E)) \hookrightarrow$ $A_{d+s-2}(\mathbf{P}(E))$ the first Chern class of a line bundle on $\mathbf{P}(E)$ is exactly its isomorphism class in the Picard group $\operatorname{Pic}(\mathbf{P}(E))$. Therefore

$s \cdot\left[O_{\mathbf{P}(E)}(1)\right]=\left[\pi^{*} L_{1}\right]+\cdots+\left[\pi^{*} L_{s}\right]+\left[D_{1}^{\prime}\right]+\cdots+\left[D_{s}^{\prime}\right]=s \cdot\left(\left[\pi^{*} L_{2}\right]+\left[D_{2}^{\prime}\right]\right)$.

It can be easily checked that $D_{2}^{\prime}$ is a Cartier divisor on $\mathbf{P}(E)$. Hence all classes $\left[O_{\mathbf{P}(E)}(1)\right],\left[\pi^{*} L_{2}\right]$ and $\left[D_{2}^{\prime}\right]$ lie in the Picard group $\operatorname{Pic}(\mathbf{P}(E))$. But this group is free abelian, because $\mathbf{P}(E)$ is complete. So the above equality is divisible by $s:\left[O_{\mathbf{P}(E)}(1)\right]=\left[\pi^{*} L_{2}\right]+\left[D_{2}^{\prime}\right]=\operatorname{deg}(F)$.

From now on we assume that $\mathbf{P}=\mathbf{P}_{\Sigma}$ is a complete simplicial toric variety and that $\operatorname{deg}\left(f_{j}\right) \in \operatorname{Pic}(\mathbf{P}), j=1, \ldots, s$. Denote by $Y$ the hypersurface in $\mathbf{P}(E)$ defined by $F=\sum_{j=1}^{s} y_{j} f_{j}$.

Lemma 2.2. $X=X_{f_{1}} \cap \ldots \cap X_{f_{s}}$ is a quasi-smooth intersection iff the hypersurface $Y$ is quasi-smooth.

Proof. $X=X_{f_{1}} \cap \ldots \cap X_{f_{s}}$ is a quasi-smooth intersection means that whenever $x \in \mathbf{V}\left(f_{1}, \ldots, f_{s}\right) \backslash Z(\Sigma)$, the $\operatorname{rank}\left(\frac{\partial f_{j}}{\partial x_{i}}(x)\right)_{i, j}=s$. And $Y$ is quasismooth iff $z=(x, y) \in \mathbf{V}(F) \backslash Z(\tilde{\Sigma})$ implies that one of the partial derivatives $\frac{\partial F}{\partial y_{j}}(z)=f_{j}(x), j=1, \ldots, s, \frac{\partial F}{\partial x_{i}}(z)=\sum_{j=1}^{s} y_{j} \frac{\partial f_{j}}{\partial x_{i}}(x), i=1, \ldots, n$, is nonzero.

So let $(x, y) \in \mathbf{V}(F) \backslash Z(\tilde{\Sigma})$, then there is a cone $\tilde{\sigma}+\sigma^{\prime} \in \tilde{\Sigma}$ with $\sigma \in \Sigma$, $\sigma^{\prime} \in \Sigma^{\prime}$, such that $\prod_{\tilde{e}_{i} \notin \tilde{\sigma}} x_{i} \prod_{\tilde{n}_{j} \notin \sigma^{\prime}} y_{j} \neq 0$ where $x_{i}, y_{j}$ are the coordinates of $(x, y)$. If $f_{1}(x)=\cdots=f_{s}(x)=0$, then $x \in \mathbf{V}\left(f_{1}, \ldots, f_{s}\right) \backslash Z(\Sigma)$ because $\prod_{e_{i} \in \sigma} x_{i} \neq 0$. And if $X=X_{f_{1}} \cap \ldots \cap X_{f_{s}}$ is a quasi-smooth intersection, one of the partial derivatives $\frac{\partial F}{\partial x_{i}}(z)=\sum_{j=1}^{s} y_{j} \frac{\partial f_{j}}{\partial x_{i}}(x), i=1, \ldots, n$, is nonzero.

Conversely, suppose $Y$ is quasi-smooth. Pick any $x \in \mathbf{V}\left(f_{1}, \ldots, f_{s}\right) \backslash$ $Z(\Sigma)$, then $(x, y) \in \mathbf{V}(F) \backslash Z(\tilde{\Sigma})$ for each $y=\left(y_{1}, \ldots, y_{s}\right) \neq 0$. Therefore $\sum_{j=1}^{s} y_{j} \frac{\partial f_{j}}{\partial x_{i}}(x) \neq 0$ for some $i$, which means the $\operatorname{rank}\left(\frac{\partial f_{j}}{\partial x_{i}}(x)\right)_{i, j}$ is maximal. 


\section{Cohomology of quasi-smooth intersections.}

Since a quasi-smooth intersection is a compact V-manifold (Proposition 1.3), the cohomology on it has a pure Hodge structure. Using Proposition 1.4 and the Poincaré duality, we can compute the cohomology of a quasi-smooth intersection except for the cohomology in the middle dimension $d-s$. So we introduce the following definition.

Definition 3.1. The variable cohomology group $H_{\mathrm{var}}^{d-s}(X)$ is $\operatorname{coker}\left(H^{d-s}(\mathbf{P})\right.$ $\left.\stackrel{i^{*}}{\rightarrow} H^{d-s}(X)\right)$.

The variable cohomology group also has a pure Hodge structure.

Proposition 3.2. Let $X=X_{f_{1}} \cap \ldots \cap X_{f_{s}}$ be a quasi-smooth intersection of ample hypersurfaces. Then there is an exact sequence of mixed Hodge structures

$$
0 \rightarrow H^{d-s-1}(\mathbf{P}) \stackrel{\cup[X]}{\rightarrow} H^{d+s-1}(\mathbf{P}) \rightarrow H^{d+s-1}(\mathbf{P} \backslash X) \rightarrow H_{\mathrm{var}}^{d-s}(X) \rightarrow 0,
$$

where $[X] \in H^{2 s}(\mathbf{P})$ is the cohomology class of $X$.

Proof. Consider the Gysin exact sequence:

$$
\cdots \rightarrow H^{i-2 s}(X) \stackrel{i_{!}}{\rightarrow} H^{i}(\mathbf{P}) \rightarrow H^{i}(\mathbf{P} \backslash X) \rightarrow H^{i-2 s+1}(X) \stackrel{i_{1}}{\rightarrow} H^{i+1}(\mathbf{P}) \rightarrow \cdots
$$

Since $i^{*}$ is Poincaré dual to the Gysin map $i_{\text {! }}$, it follows that $H_{\mathrm{var}}^{d-s}(X)$ is isomorphic to the kernel of $i_{!}: H^{d-s}(X) \rightarrow H^{d+s}(P)$. So we get an exact sequence

$$
H^{d-s-1}(X) \stackrel{i_{!}}{\rightarrow} H^{d+s-1}(\mathbf{P}) \rightarrow H^{d+s-1}(\mathbf{P} \backslash X) \rightarrow H_{\mathrm{var}}^{d-s}(X) \rightarrow 0 .
$$

Now we use a commutative diagram

$$
\begin{array}{ll}
H^{d-s-1}(X) & \stackrel{i !}{\rightarrow} H^{d+s-1}(\mathbf{P}) \\
\stackrel{i^{*} \uparrow}{ } & \nearrow \cup[X] \\
H^{d-s-1}(\mathbf{P}) . &
\end{array}
$$

By Proposition $1.4 i^{*}$ is an isomorphism in this diagram, so it suffices to prove that the Gysin map $i_{\text {! }}$ is injective in the above diagram.

Lemma 3.3. If $X=X_{f_{1}} \cap \ldots \cap X_{f_{s}}$ is a quasi-smooth intersection of ample hypersurfaces, then the Gysin map $H^{d-s-1}(X) \stackrel{i_{1}}{\rightarrow} H^{d+s-1}(\mathbf{P})$ is injective.

Proof. Since the odd dimensional cohomology of a complete simplicial toric variety vanishes $\left[\mathbf{F 1}\right.$, pp. 92-94] and $i^{*}: H^{d-s-1}(\mathbf{P}) \rightarrow H^{d-s-1}(X)$ is an isomorphism by Proposition 1.4, it follows that $H^{d-s-1}(X)=H^{d-s-1}(\mathbf{P})=$ $H^{d+s-1}(\mathbf{P})=0$ when $d+s-1$ is odd. So by the Gysin exact sequence (1) it is enough to show that $H^{d+s-2}(\mathbf{P} \backslash X)=0$ when $d+s-2$ is odd. To prove this we use the Cayley trick again. Let $Y$ be the hypersurface defined by 
$F=\sum_{j=1}^{s} y_{j} f_{j}$. Then the natural map $\mathbf{P}(E) \backslash Y \rightarrow \mathbf{P} \backslash X$, induced by the projection $\pi: \mathbf{P}(E) \rightarrow \mathbf{P}$, is a $\mathbf{C}^{s-1}$ bundle in the Zariski topology. Notice that $\mathbf{P} \backslash X$ is simply connected, because $\mathbf{P}$ is simply connected $[\mathbf{F} \mathbf{1}$, p. 56] and $X$ has codimension at least 2 in $\mathbf{P}$. Hence, the Leray-Serre spectral sequence implies that $H^{i}(\mathbf{P}(E) \backslash Y)=H^{i}(\mathbf{P} \backslash X)$ for $i \geq 0$. We have that $H^{d+s-2}(\mathbf{P}(E))=0$ for $d+s-2$ odd and $Y$ is quasi-smooth by Lemma 2.2. So from the Gysin exact sequence

$$
H^{d+s-2}(\mathbf{P}(E)) \rightarrow H^{d+s-2}(\mathbf{P}(E) \backslash Y) \rightarrow H^{d+s-3}(Y) \stackrel{j !}{\rightarrow} H^{d+s-1}(\mathbf{P}(E))
$$

(here the Gysin map $j$ ! is induced by the inclusion $j: Y \hookrightarrow \mathbf{P}(E)$ ) it follows that we need to show injectivity of $j_{!}: H^{d+s-3}(Y) \rightarrow H^{d+s-1}(\mathbf{P}(E))$. Consider the commutative diagram

$$
\begin{array}{cl}
H^{d+s-3}(Y) & \stackrel{j !}{\rightarrow} H^{d+s-1}(\mathbf{P}(E)) \\
H^{*} \uparrow & \nearrow \cup[Y] \\
H^{d+s-3}(\mathbf{P}(E)) &
\end{array}
$$

where $[Y] \in H^{2}(\mathbf{P}(E))$ is the cohomology class of $Y$. The canonical line bundle $O_{\mathbf{P}(E)}(1)$ is ample [H, III, §1], whence by Lemma 2.1, $Y$ is ample. So by Proposition $10.8[\mathbf{B C}] j^{*}: H^{d+s-3}(\mathbf{P}(E)) \rightarrow H^{d+s-3}(Y)$ is an isomorphism and by Hard Lefschetz $\cup[Y]: H^{d+s-3}(\mathbf{P}(E)) \rightarrow H^{d+s-1}(\mathbf{P}(E))$ is injective. Thus, from the above diagram the lemma follows.

Definition 3.4. For a nonzero polynomial $F \in R=\mathbf{C}\left[x_{1}, \ldots, x_{n}, y_{1}, \ldots, y_{s}\right]$ the Jacobian ring $R(F)$ denotes the quotient of $R$ by the ideal generated by the partial derivatives $\frac{\partial F}{\partial y_{j}}, j=1, \ldots, s, \frac{\partial F}{\partial x_{i}}, i=1, \ldots, n$.

Remark 3.5. If $F=y_{1} f_{1}+\cdots+y_{s} f_{s}$ is as in Lemma 2.1 with $f_{j} \in S_{\alpha_{j}}$, then $R(F)$ carries a natural grading by the Chow group $A_{d+s-2}(\mathbf{P}(E))$. Moreover, there are canonical isomorphisms $A_{d+s-2}(\mathbf{P}(E)) \cong A_{d-1}(\mathbf{P}) \oplus A_{d}(\mathbf{P}) \cong$ $A_{d-1}(\mathbf{P}) \oplus \mathbf{Z}([\mathbf{F 2}])$. With respect to this bigrading of the Chow group $A_{d+s-2}(\mathbf{P}(E))$ we have that $\operatorname{deg}(F)=(0,1), \operatorname{deg}\left(f_{j}\right)=\left(\alpha_{j}, 0\right), \operatorname{deg}\left(y_{j}\right)=$ $\left(-\alpha_{j}, 1\right)$, which is very similar to the case when $\mathbf{P}$ is a projective space.

We now can state the main result.

Theorem 3.6. Let $\mathbf{P}$ be a d-dimensional complete simplicial toric variety, and let $X \subset \mathbf{P}$ be a quasi-smooth intersection of ample hypersurfaces defined by $f_{j} \in S_{\alpha_{j}}, j=1, \ldots, s$. If $F=y_{1} f_{1}+\cdots+y_{s} f_{s}$, then for $p \neq \frac{d+s-1}{2}$, we have a canonical isomorphism

$$
R(F)_{(d+s-p) \beta-\beta_{0}} \cong H_{\mathrm{var}}^{p-s, d-p}(X)
$$

where $\beta_{0}=\operatorname{deg}\left(x_{1} \cdots x_{n} \cdot y_{1} \cdots y_{s}\right), \beta=\operatorname{deg}(F)=\operatorname{deg}\left(f_{j}\right)+\operatorname{deg}\left(y_{j}\right)$. In the case $p=\frac{d+s-1}{2}$ there is an exact sequence

$0 \rightarrow H^{d-s-1}(\mathbf{P}) \stackrel{\cup[X]}{\rightarrow} H^{d+s-1}(\mathbf{P}) \rightarrow R(F)_{\frac{d+s+1}{2} \beta-\beta_{0}} \rightarrow H_{\mathrm{var}^{2}}^{\frac{d-s-1}{2}}, \frac{d-s+1}{2}(X) \rightarrow 0$. 
Proof. Since $H^{i}(\mathbf{P})$ vanishes for $i$ odd and has a pure Hodge structure of type $(p, p)$ for $i$ even, from Proposition 3.2 we get $\operatorname{Gr}_{F}^{p} H^{d+s-1}(\mathbf{P} \backslash X) \cong$ $H_{v a r}^{p-s, d-p}(X)$ if $p \neq \frac{d+s-1}{2}$, and in case $p=\frac{d+s-1}{2}$ the following sequence

$$
\begin{aligned}
0 \rightarrow H^{d-s-1}(\mathbf{P}) \stackrel{\cup[X]}{\rightarrow} & H^{d+s-1}(\mathbf{P}) \\
& \rightarrow \operatorname{Gr}_{F}^{\frac{d+s-1}{2}} H^{d+s-1}(\mathbf{P} \backslash X) \rightarrow H_{v a r}^{\frac{d-s-1}{2}}, \frac{d-s+1}{2}(X) \rightarrow 0
\end{aligned}
$$

is exact.

Now use the isomorphism of mixed Hodge structures $H^{i}(\mathbf{P} \backslash X) \cong$ $H^{i}(\mathbf{P}(E) \backslash Y)$ and by the Theorem $10.6[\mathbf{B C}]$ the desired result follows.

\section{Cohomology of nondegenerate intersections.}

In this section we consider a special case of quasi-smooth intersections.

Definition 4.1. A closed subset $X=X_{f_{1}} \cap \ldots \cap X_{f_{s}}$, defined by D-homogeneous polynomials $f_{1}, \ldots, f_{s}$, is called a nondegenerate intersection if $X_{f_{j_{1}}} \cap$ $\ldots \cap X_{f_{j_{k}}} \cap \mathbf{T}_{\tau}$ is a smooth subvariety of codimension $k$ in $\mathbf{T}_{\tau}$ for any $\left\{j_{1}, \ldots, j_{k}\right\} \subset\{1, \ldots, s\}$ and $\tau \in \Sigma$. (Here $\mathbf{T}_{\tau}$ denotes the torus in $\mathbf{P}_{\Sigma}$ associated with a cone $\tau \in \Sigma$.)

We will show how to define a nondegenerate intersection in terms of the polynomials $f_{1}, \ldots, f_{s}$. For $\sigma \in \Sigma$, let $U_{\sigma}=\left\{x \in \mathbf{A}^{n}: \hat{x}_{\sigma} \neq 0\right\}$. We know that $\mathbf{P}_{\Sigma}$ has an affine toric open cover by $\mathbf{A}_{\sigma}=U_{\sigma} / \mathbf{D}(\Sigma), \sigma \in \Sigma[\mathbf{B C}]$. Also $\mathbf{T}_{\tau}=\left(U_{\tau} \backslash \cup_{\gamma \prec \tau} U_{\gamma}\right) / \mathbf{D}(\Sigma)$. Notice that $U_{\tau} \backslash \cup_{\gamma \prec \tau} U_{\gamma}=\left\{x \in \mathbf{A}^{n}\right.$ : $\hat{x}_{\tau} \neq 0, x_{i}=0$ if $\left.\rho_{i} \subset \tau\right\}$ is a torus. So each $\mathbf{T}_{\tau}$ is a quotient of a torus by a $D$-subgroup, because $\mathbf{D}$ is diagonalizable $[\mathbf{B C}]$.

Lemma 4.2. Let $T=\left(\mathbf{C}^{*}\right)^{n} / G$ be the quotient of a torus by a D-subgroup $G$. Suppose that $X \subset\left(\mathbf{C}^{*}\right)^{n}$ is an invariant subvariety with respect to the action of $G$. Then the geometric quotient $X / G$ is smooth iff $X$ is smooth.

Proof. By the structure theorem of a $D$-group $[\mathbf{H u}, \S 16.2]$ we can assume that $\left(\mathbf{C}^{*}\right)^{n}=G^{\circ} \times\left(\mathbf{C}^{*}\right)^{k}$, where $G^{\circ} \cong\left(\mathbf{C}^{*}\right)^{n-k}$ is the identity component of $G$, and $G=G^{\circ} \times H$ for some finite subgroup $H$ in $\left(\mathbf{C}^{*}\right)^{k}$. Now it suffices to show the Lemma if $G$ is a torus or a finite group. If $G=G^{\circ}$ then $X=\left(\mathbf{C}^{*}\right)^{n-k} \times p(X)$, where by $p(X)$ we mean the projection of $X$ onto $\left(\mathbf{C}^{*}\right)^{k}$. Notice that $p(X) \cong X / G$, hence $X$ is smooth iff $X / G$ is smooth. In the case $G=H$ is a finite group it can be easily checked that $X \rightarrow$ $X / G$ is an unramified cover [Sh, p. 346]. So $X$ and $X / G$ are smooth simultaneously.

From this Lemma it follows that $X=X_{f_{1}} \cap \ldots \cap X_{f_{s}}$ is a nondegenerate intersection iff $\mathbf{V}\left(f_{j_{1}}, \ldots, f_{j_{k}}\right) \cap V_{\tau}$ is a smooth subvariety of codimension $k$ in the torus $V_{\tau}=\left\{x \in \mathbf{A}^{n}: \hat{x}_{\tau} \neq 0, x_{i}=0\right.$ if $\left.\rho_{i} \subset \tau\right\}$. 
As in Section 2 we can consider the hypersurface $Y \subset \mathbf{P}(E)$ defined by $F=\sum_{j=1}^{s} y_{j} f_{j}$.

Lemma 4.3. $X=X_{f_{1}} \cap \ldots \cap X_{f_{s}}$ is a nondegenerate intersection iff $Y$ is a nondegenerate hypersurface.

Proof. As shown above, $X=X_{f_{1}} \cap \ldots \cap X_{f_{s}}$ is a nondegenerate intersection if the $\operatorname{rank}\left(\frac{\partial f_{j}}{\partial x_{i}}(x)\right)_{i \in\left\{i: e_{i} \notin \tau\right\}}^{j \in\left\{j_{1}, \ldots, j_{k}\right\}}=k$ for all $x \in \mathbf{V}\left(f_{j_{1}}, \ldots, f_{j_{k}}\right) \cap V_{\tau}, \tau \in \Sigma$ and $\left\{j_{1}, \ldots, j_{k}\right\} \subset\{1, \ldots, s\}$. Similarly $Y$ is nondegenerate iff $z=(x, y) \in$ $\mathbf{V}(F) \cap V_{\tilde{\tau}+\tau^{\prime}}, \tilde{\tau}+\tau^{\prime} \in \tilde{\Sigma}$ with $\tau \in \Sigma, \tau^{\prime} \in \Sigma^{\prime}$ (recall the definition of $\mathbf{P}(E)$ associated with $\tilde{\Sigma}$ in the Section 2) implies that one of the partial derivatives $\frac{\partial F}{\partial y_{j}}(z)=f_{j}(x), j \in\left\{j: \tilde{n}_{j} \notin \tau^{\prime}\right\}, \frac{\partial F}{\partial x_{i}}(z)=\sum_{j=1}^{s} y_{j} \frac{\partial f_{j}}{\partial x_{i}}(x), i \in\left\{i: \tilde{e}_{i} \notin \tilde{\tau}\right\}$, is nonzero.

Let $(x, y) \in \mathbf{V}(F) \cap V_{\tilde{\tau}+\tau^{\prime}}$, where $\tilde{\tau}+\tau^{\prime} \in \tilde{\Sigma}$ with $\tau \in \Sigma, \tau^{\prime} \in \Sigma^{\prime}$. Then $\prod_{\tilde{e}_{i} \notin \tilde{\tau}} x_{i} \prod_{\tilde{n}_{j} \notin \tau^{\prime}} y_{j} \neq 0$ and $x_{i}=0$ if $\tilde{e}_{i} \in \tilde{\tau}, y_{j}=0$ if $\tilde{n}_{j} \in \tau^{\prime}$. If $f_{j}(x)=0$ for all $j \in\left\{j: \tilde{n}_{j} \notin \tau^{\prime}\right\}$, then $x \in \mathbf{V}\left(f_{j_{1}}, \ldots, f_{j_{k}}\right) \cap V_{\tau}$ where $\left\{j_{1}, \ldots, j_{k}\right\}=$ $\left\{j: \tilde{n}_{j} \notin \tau^{\prime}\right\}$. So if $X=X_{f_{1}} \cap \ldots \cap X_{f_{s}}$ is a nondegenerate intersection, one of the partial derivatives $\frac{\partial F}{\partial x_{i}}(z)=\sum_{j=1}^{s} y_{j} \frac{\partial f_{j}}{\partial x_{i}}(x), i \in\left\{i: \tilde{e}_{i} \notin \tilde{\tau}\right\}$, is nonzero.

Conversely, suppose $Y$ is nondegenerate. Take any $x \in \mathbf{V}\left(f_{j_{1}}, \ldots, f_{j_{k}}\right) \cap V_{\tau}$ with $\tau \in \Sigma,\left\{j_{1}, \ldots, j_{k}\right\} \subset\{1, \ldots, s\}$. Then $(x, y) \in \mathbf{V}(F) \cap V_{\tilde{\tau}+\tau^{\prime}}$ for each $y \in V_{\tau^{\prime}}=\left\{y \in \mathbf{A}^{s}: y_{j} \neq 0\right.$ if $\tilde{n}_{j} \notin \tau^{\prime}, y_{j}=0$ if $\left.\tilde{n}_{j} \in \tau^{\prime}\right\}$ where $\tau^{\prime}$ is the cone generated by the complement of $\left\{\tilde{n}_{j_{1}}, \ldots, \tilde{n}_{j_{k}}\right\}$ in the set $\left\{\tilde{n}_{1}, \ldots, \tilde{n}_{s}\right\}$. Therefore $\sum_{j=1}^{s} y_{j} \frac{\partial f_{j}}{\partial x_{i}}(x) \neq 0$ for some $i$, which means the $\operatorname{rank}\left(\frac{\partial f_{j}}{\partial x_{i}}(x)\right)_{i \in\left\{i: e_{i} \notin \tau\right\}}^{j \in\left\{j_{1}, \ldots, j_{k}\right\}}=k$.

Since a nondegenerate hypersurface is quasi-smooth [BC], Lemma 2.2 shows that a nondegenerate intersection is quasi-smooth.

Definition 4.4 ([BC]). Given a polynomial $f \in S=\mathbf{C}\left[x_{1}, \ldots, x_{n}\right]$, we get the ideal quotient $J_{1}(f)=\left\langle x_{1} \partial f / \partial x_{1}, \ldots, x_{n} \partial f / \partial x_{n}\right\rangle: x_{1} \cdots x_{n}$ (see [CLO, p. 193]) and the ring $R_{1}(f)=S / J_{1}(f)$.

Remark 4.5. If $F=\sum_{j=1}^{s} y_{j} f_{j} \in R$ is as in Lemma 2.1, then $R_{1}(F)=$ $R / J_{1}(F)$ has a natural grading by the Chow group $A_{d+s-2}(\mathbf{P}(E)) \cong$ $A_{d-1}(\mathbf{P}) \oplus \mathbf{Z}$.

Theorem 4.6. Let $X=X_{f_{1}} \cap \ldots \cap X_{f_{s}}$ be a nondegenerate intersection of ample hypersurfaces given by $f_{j} \in S_{\alpha_{j}}, j=1, \ldots, s$. If $F=\sum_{j=1}^{s} y_{j} f_{j} \in R$, then there is a canonical isomorphism

$$
H_{\mathrm{var}}^{p-s, d-p}(X)=R_{1}(F)_{(d+s-p) \beta-\beta_{0}},
$$

where $\beta_{0}=\operatorname{deg}\left(x_{1} \cdots x_{n} \cdot y_{1} \cdots y_{s}\right), \beta=\operatorname{deg}(F)$. 
Proof. First we will show that there is an isomorphism of Hodge structures $H_{\text {var }}^{d-s}(X)(1-s) \cong H_{\mathrm{var}}^{d+s-2}(Y)$. Let $\varphi: Y \rightarrow \mathbf{P}$ be the composition of the inclusion $j: Y \hookrightarrow \mathbf{P}(E)$ and the projection $\pi: \mathbf{P}(E) \rightarrow \mathbf{P}$. As in [Te], consider the following morphism of the Leray spectral sequences

$$
\begin{array}{ccccc}
E_{2}^{p, q}= & H^{p}\left(\mathbf{P}, R^{q} \pi_{*} \mathbf{C}\right) & \Rightarrow & H^{p+q}(\mathbf{P}(E)) \\
\downarrow & & & \downarrow \\
{ }^{\prime} E_{2}^{p, q}= & H^{p}\left(\mathbf{P}, R^{q} \varphi_{*} \mathbf{C}\right) & \Rightarrow & H^{p+q}(Y) .
\end{array}
$$

Since

$$
\varphi^{-1}(X)= \begin{cases}\mathbb{P}^{s-1} & \text { if } x \in X \\ \mathbb{P}^{s-2} & \text { if } x \notin X,\end{cases}
$$

we have that (see [Go, p. 202], [De])

$$
R^{q} \varphi_{*} \mathbf{C}= \begin{cases}\mathbf{C}_{\mathbf{P}}\left(-\frac{q}{2}\right) & \text { if } q \text { is even and } 0 \leq q<2 s-2 \\ \mathbf{C}_{X}(1-s) & \text { if } q=2 s-2 \\ 0 & \text { otherwise }\end{cases}
$$

Also we have

$$
R^{q} \pi_{*} \mathbf{C}=\left\{\begin{array}{cl}
\mathbf{C}_{\mathbf{P}}\left(-\frac{q}{2}\right) & \text { if } q \text { is even and } 0 \leq q \leq 2 s-2, \\
0 & \text { otherwise. }
\end{array}\right.
$$

The first spectral sequence degenerates at $E_{2}$, because for $p$ or $q$ odd $E_{r}^{p, q}$ vanishes. The second spectral sequence also degenerates at $E_{2}$ :

$$
h^{l-2 s-2}(X)+\sum_{q=0}^{2 s-4} h^{l-q}(\mathbf{P})=\sum_{p+q=l} \operatorname{dim}^{\prime} E_{2}^{p, q} \geq \sum_{p+q=l} \operatorname{dim}^{\prime} E_{\infty}^{p, q}=h^{l}(Y) .
$$

To show the degeneracy of ${ }^{\prime} E_{2}^{p, q}$ it suffices to show that the above inequality is an equality. From Proposition 10.8 [BC] and Proposition 3.2 we get

$$
\begin{gathered}
h^{d+s-2}(Y)=h^{d+s-2}(\mathbf{P}(E))+h^{d+s-1}(\mathbf{P}(E) \backslash Y) \\
\quad-h^{d+s-1}(\mathbf{P}(E))+h^{d+s-3}(\mathbf{P}(E)), \\
h^{d-s}(X)=h^{d-s}(\mathbf{P})+h^{d+s-1}(\mathbf{P} \backslash X)-h^{d+s-1}(\mathbf{P})+h^{d-s-1}(\mathbf{P}) .
\end{gathered}
$$

Hence, using the spectral sequence $E_{2}^{p, q}$, we can easily compute the Hodge numbers of $\mathbf{P}(E)$ and check that $h^{l-2 s-2}(X)+\sum_{q=0}^{2 s-4} h^{l-q}(\mathbf{P})=h^{l}(Y)$ for $l=d+s-2$. Using Proposition 1.4, we can similarly show the above equality for $l \neq d+s-2$ as well. So the spectral sequence ' $E_{2}^{p, q}$ degenerates at $E_{2}$. Since $E_{2}^{d+s-2-q, q}={ }^{\prime} E_{2}^{d+s-2-q, q}$ for $q \neq 2 s-2$ and, by Proposition $1.4, E_{2}^{d-s, 2 s-2} \hookrightarrow{ }^{\prime} E_{2}^{d-s, 2 s-2}$, we get an isomorphism of Hodge structures (for details see $[\mathbf{T e}]$ ):

$$
H_{v a r}^{d+s-2}(Y) \cong{ }^{\prime} E_{2}^{d-s, 2 s-s} / E_{2}^{d-s, 2 s-2} \cong H_{v a r}^{d-s}(X)(1-s) .
$$

Now we only need to apply Theorem $11.8[\mathbf{B C}]$ to finish the proof. 


\section{References}

[BB] V.V. Batyrev and L. Borisov, Dual cones and mirror symmetry for generalized Calabi-Yau manifolds, in Mirror Symmetry II, Ed. B. Greene and S.-T. Yau, AMSIP, Providence, 1996.

[BC] V.V. Batyrev and D.A. Cox, On the Hodge structure of projective hypersurfaces in toric varieties, Duke Math. J., 75 (1994), 293-338.

[CCD] E. Cattani, D. Cox and A. Dickenstein, Residues in toric varieties, Compositio Mathematica, 108 (1997), 35-76.

[C] D. Cox, The homogeneous coordinate ring of a toric variety, J. Algebraic Geom., 4 (1995), 17-50.

[CLO] D. Cox, J. Little and D. O'Shea, Ideals, Varieties and Algorithms, Springer-Verlag, New-York, 1992.

[Da] V. Danilov, The geometry of toric varieties, Russian Math. Surveys, 33 (1978), 97-154.

[DK] V. Danilov and A. Khovanskii, Newton polyhedra and an algorithm for computing Hodge-Deligne numbers, Math. USSR-Izv., 29 (1987), 279-298.

[De] P. Deligne, Théorie de Hodge, II, III, Inst. Hautes Études Sci. Publ. Math., 40 (1971), 5-58; 44 (1975), 5-77.

[Di] A. Dimca, Residues and cohomology of complete intersections, Duke Math. J., 78 (1995), 89-100.

[Dol] I. Dolgachev, Weighted projective varieties, in 'Lecture Notes in Math.', 956, Springer-Verlag, Berlin, (1982), 34-71.

[F1] W. Fulton, Introduction to Toric Varieties, Princeton Univ. Press, Princeton, NJ, 1993.

[F2] , Intersection Theory, Springer-Verlag, Berlin, 1984.

[FH] W. Fulton and J. Hansen, A connectedness theorem for projective varieties, with applications to intersections and singularities of mappings, Annals of Math., 110 (1979), 159-166.

[FL1] W. Fulton and R. Lazarsfeld, On the connectedness of degeneracy loci and special divisors, Acta Math., 146 (1981), 271-283.

[FL2] _ Connectivity and its Applications in Algebraic Geometry, Lecture Notes in Math., 862, Springer-Verlag, Berlin-Heidelberg, (1981), 26-92.

[GKZ] I. Gelfand, M. Kapranov and A. Zelevinsky, Discriminants, Resultants, and Multidimensional Determinants, Birkhäuser Verlag, Basel-Boston, 1994.

[Go] R. Godement, Topologie Algébrique et Théorie des Faiceaux, Hemann, Paris, 1958.

[Gr] P. Griffiths, On the periods of certain rational integrals, I, II, Ann. of Math., 90(2) (1969), 460-495, 498-541.

[H] R. Hartshorne, Ample Subvarieties of Algebraic Varieties, Lecture Notes in Math., 156, Springer-Verlag, Berlin-Heidelberg, 1970.

[Hu] J. Humphreys, Linear Algebraic Groups, Springer-Verlag, Berlin, 1977.

[Ko] K. Konno, On the variational Torelli problem for complete intersections, Comp. Math., 78 (1991), 271-296.

[L] A. Libgober, Differential forms on complete intersections and related quotient module, Israel Mathematical Conference Proceedings, 9 (1996), 295-305. 
[M] Yu. Manin, Lectures on the K-functor in algebraic geometry, Russ. Math. Surveys, 24(5) (1969), 1-89.

[Na] J. Nagel, The Abel-Jacobi map for complete intersections, Indag. Math., 8 (1997), 95-113.

[O] T. Oda, Convex Bodies and Algebraic Geometry, Springer-Verlag, Berlin, 1988.

[PS] C. Peters and J. Steenbrink, Infinitesimal Variations of Hodge Structure and the Generic Torelli Problem for Projective Hypersurfaces (after Carlson, Donagi, Green, Griffiths, Harris), Classification of Algebraic and Analytic Manifolds, Ed. K. Ueno, Progr. Math., 39, Birkhäuser, Boston, (1983), 399-463.

[Sh] I.R. Shafarevich, Basic Algebraic Geometry, Springer-Verlag, New York-Berlin, 1974.

[St] J. Steenbrink, Intersection form for quasi-homogeneous singularities, Compositio Math., 34 (1977), 211-223.

[Te] T. Terasoma, Infinitesimal variation of Hodge structures and the weak global Torelli theorem for complete intersections, Ann. of Math., 132 (1990), 213-235.

Received February 18, 1998.

University of Massachusetts

AmHerst, MA 01003

E-mail address: anvar@math.umass.edu 